\title{
A Study of Human Chorionic Gonadotropin in the Pathogenesis of Hypertensive Disorders of Pregnancy
}

\author{
${ }^{1}$ Priyadarshini Tiwari, ${ }^{2}$ Pooja Saraogi, ${ }^{3}$ Sonal Sahni, ${ }^{4}$ Bharti Sahu, ${ }^{5}$ Poorva Badkur
}

\begin{abstract}
Objectives: The aim of our study was to observe the levels of serum beta-human chorionic gonadotropin ( $\beta$-hCG) in cases of preeclampsia and eclampsia in the 3rd trimester of pregnancy and to compare it with the level of serum $\beta$-hCG in normotensive controls and also to find out if any correlation exists between the levels of $\beta-\mathrm{hCG}$ and the severity of the disease.
\end{abstract}

Materials and methods: A case-control study of 130 cases of pregnancy-induced hypertension (PIH), out of which 100 were cases of preeclampsia and 30 were those of eclampsia, was conducted with 100 normotensive antenatal cases as controls. Patients admitted as preeclampsia or eclampsia were subjected for the study of $\beta$-hCG from 28 weeks onwards depending on the gestational age at the time of admission. Normotensive patients coming as outpatients were taken as controls and their $\beta$-hCG estimation was done.

Results: The $\beta$-hCG levels in hypertensive patients were found to be significantly higher than that of the controls. There was a significant correlation with the severity of the disease. A significant positive correlation was found with the systolic BP, diastolic $\mathrm{BP}$, alanine transaminase, aspartate transaminase, and serum bilirubin whereas a significant inverse correlation was found with high-density lipoprotein (HDL) and platelet counts.

Conclusion: Beta-human chorionic gonadotropin rises significantly in the cases of $\mathrm{PIH}$ and the rise correlates with the severity of hypertension and the derangement of laboratory parameters. As it has a low sensitivity and specificity, it is not a very useful tool as a diagnostic or prognostic indicator for preeclampsia.

Keywords: Beta-human chorionic gonadotropin, Eclampsia, Preeclampsia.

How to cite this article: Tiwari P, Saraogi P, Sahni S, Sahu B, Badkur P. A Study of Human Chorionic Gonadotropin in the Pathogenesis of Hypertensive Disorders of Pregnancy. J South Asian Feder Obst Gynae 2016;8(4):282-285.

Source of support: Nil

Conflict of interest: None

Date of received: 27 May 2016

Date of acceptance: 17 September 2016

Date of publication: October 2016

\footnotetext{
1,3,4 Assistant Professor, ${ }^{2}$ Senior Resident, ${ }^{5}$ Registrar

${ }^{1-5}$ Department of Obstetrics and Gynecology, Netaji Subhash Chandra Bose Medical College \& Hospital, Jabalpur, Madhya Pradesh, India

Corresponding Author: Priyadarshini Tiwari, Assistant Professor Department of Obstetrics and Gynecology, Netaji Subhash Chandra Bose Medical College \& Hospital, Jabalpur, Madhya Pradesh, India e-mail: drpriya2004@yahoo.co.in
}

\section{INTRODUCTION}

Preeclampsia is characterized by hypertension in pregnancy along with proteinurea. ${ }^{1}$ When there is onset of convulsions in cases of preeclampsia, it is termed as eclampsia. Hypertensive disorder of pregnancy is basically a microangiopathy with endothelial damage and hypoxic injury in the placenta. ${ }^{2}$ It can be said that eclampsia also forms a part of the continuum of disease which starts from mild preeclampsia and progresses to severe preeclampsia and eclampsia.

A normal pregnancy is characterized by trophoblastic invasion of spiral arterioles in 1st trimester as well as 2nd trimester. In the 1st trimester there is invasion of the decidual segment of spiral arterioles, whereas in the 2nd trimester there is invasion of myometrial segment of spiral arterioles by cytotrophoblast. As a result of this, the decidual as well as myometrial segment of the spiral arterioles lose their smooth muscle coat and become unresponsive to the effect of circulating pressor agents.

In cases of preeclampsia, this 2 nd wave of invasion fails to occur ${ }^{3}$ and the spiral arterioles remain thick-walled and responsive to pressor substances, leading to a higher resistance in the placental bed. ${ }^{4,5}$ This pathology ultimately leads to hypoxia in the placental bed, ${ }^{6}$ leading to free radical-mediated injury in the vessels of placenta. This has multiple manifestations, namely maternal hypertension and fetal intrauterine growth restriction. Extreme cases progress to a microangiopathy called HELLP syndrome (hemolysis, elevated liver enzymes and low platelets). ${ }^{7}$

The human placenta has two kinds of trophoblast, namely the cytotrophoblast and syncytiotrophoblast. The syncytiotrophoblast is more specialized and is the source of hCG in pregnancy with high levels being achieved in the 1st trimester and the levels coming down to plateau in the 2nd trimester. When free radical-mediated injury occurs in the late 2 nd and in the 3 rd trimester, the syncytiotrophoblast is stimulated to secrete hCG which manifests as a rising level of HCG in the 3rd trimester.

\section{MATERIALS AND METHODS}

This was a case-control study conducted in Netaji Subhash Chandra Bose Medical College and Hospital (NSCB MCH) from October 2014 to 2015. The study was performed after the approval of the Ethical Committee of our institution. Written informed consent was taken from 
the patients in the study group as well as the controls. A total of 230 pregnant women were enrolled in the study. Out of these, 100 were normotensive and 130 were cases of preeclampsia and eclampsia. The cases were subgrouped into mild preeclampsia (BP $\geq 140 / 90)$, severe preeclampsia (BP $\geq 160 / 110)$, and eclampsia (preeclampsia with convulsions). The sample size was determined by simple random sampling assuming the probability of prevalence to be $17 \%$ with relative $5 \%$ of precision error. All primigravidae and multigravidae with singleton pregnancy in 3rd trimester (28 weeks and above) as determined by ultrasonography or last menstrual period were included in the study. Women with multiple pregnancy, chronic hypertension, diabetes, and fetal congenital anomalies were excluded from the study. The demographic parameters of the patients were recorded and their clinical features were noted. In the control group fasting samples of the patients were collected on outpatient basis, whereas in the study group fasting samples of admitted patients were collected.

Statistical analysis was done using Statistical Package for the Social Sciences (SPSS) software. Categorical variables were summarized in frequency and percent distribution, and chi-square or Fisher's exact test was performed as appropriate. Odds ratio with $95 \%$ confidence interval was analyzed for $2 \times 2$ distributions. Continuous variables were analyzed using mean $\pm S D$. Mean difference between two independent groups was analyzed by using independent t-test after the normalized distribution, otherwise nonparametric test was applied. One-way analysis of variance (ANOVA) with Bonferroni adjustment was also applied for comparison between more than two means. To test the null hypothesis, 0.05 Alpha and 95\% confidence limit was applied.

\section{RESULTS AND DISCUSSION}

It was observed that the members of the study population were either illiterate or educated up to middle school as against the controls that had a notably higher level of education, i.e., higher secondary and in some cases graduation showing the impact of education on antenatal care and prevention of preeclampsia and eclampsia.

There was a significantly higher number of primigravidae in the study group as compared with the controls. This higher prevalence of preeclampsia was also seen in other studies. ${ }^{8}$ Comparison of the clinical characteristics of the two groups showed a significant difference in the systolic and diastolic blood pressure $(\mathrm{p}<0.0001)$ (Table 1$)$. There was no significant difference in the hemoglobin level of the two groups. The platelets count in the study group was significantly lower as compared with the controls $(\mathrm{p}<0.0001)$. On comparing the lipid profiles in the two groups, there was no significant difference in the total cholesterol level. There was a highly significant lower level of high-density lipoprotein (HDL) $(\mathrm{p}<0.0001)$ in the study population as compared with the control. The level of low-density lipoprotein (LDL) was significantly high in the study group ( $\mathrm{p}=0.05)$.

The levels of aspartate transaminase (AST), alanine transaminase (ALT), and serum bilirubin were significantly higher in the study group $(p<0.0001),(p=0.04)$, and $(p<0.0001)$ respectively, demonstrating the hepatic microangiopathy in cases of preeclampsia leading to HELLP syndrome in the more florid cases.

The mean value of hCG was significantly higher in the study group $(\mathrm{p}<0.0001),{ }^{9}$ which also showed a significant rise according to the severity of disease, with levels being significantly higher in mild preeclampsia as compared with normotensive patients, and the levels in severe preeclampsia being significantly higher than those of mild preeclampsia $^{10}$ (Table 2, Graph 1). Paradoxically, the level of hCG in eclampsia was almost similar to that of severe preeclampsia. The levels of hCG rose with the increasing oxidative stress being higher in proportion to the severity

Table 1: comparison of baseline characteristics of the cases and controls with their statistical significance

\begin{tabular}{|c|c|c|c|c|c|c|c|c|}
\hline & \multicolumn{3}{|c|}{ Normal } & \multicolumn{3}{|c|}{ Preeclampsia and eclampsia } & \multirow[b]{2}{*}{$p$-value } & \multirow[b]{2}{*}{$t$-value } \\
\hline & $n$ & Mean $\pm S D$ & Range & $n$ & Mean $\pm S D$ & Range & & \\
\hline Age & 100 & $23.76 \pm 3.291$ & $19-35$ & 130 & $24.11 \pm 3.637$ & $19-36$ & 0.45 & 0.75 \\
\hline Systolic BP & 100 & $112 \pm 9.101$ & $70-130$ & 130 & $157.62 \pm 20.376$ & $130-240$ & $<0.0001$ & 20.84 \\
\hline Diastolic BP & 100 & $73.6 \pm 5.949$ & $60-90$ & 130 & $103 \pm 11.855$ & $90-140$ & $<0.0001$ & 22.69 \\
\hline $\mathrm{Hb}$ & 100 & $10.44 \pm 1.3729$ & $7.4-13.5$ & 130 & $10.96 \pm 6.79679$ & $6.8-86$ & 0.45 & 0.75 \\
\hline Platelets & 100 & $2.4909 \pm 0.4431$ & $1.56-3.8$ & 130 & $2.1465 \pm 0.51161$ & $0.5-3.8$ & $<0.0001$ & 8.26 \\
\hline Total cholesterol & 100 & $166.64 \pm 12.681$ & 138-194 & 130 & $181.06 \pm 48.134$ & $109-382$ & 0.004 & 2.92 \\
\hline $\mathrm{HDL}$ & 100 & $44.37 \pm 6.542$ & $30-60$ & 130 & $39.13 \pm 8.711$ & $23-68$ & $<0.0001$ & 5.03 \\
\hline LDL & 100 & $94.32 \pm 11.053$ & $74-124$ & 130 & $88.2 \pm 29.355$ & $24-232$ & 0.05 & 1.98 \\
\hline SGOT & 100 & $21.42 \pm 5.375$ & $12-34$ & 130 & $49.49 \pm 55.549$ & $14-565$ & $<0.0001$ & 5.03 \\
\hline SGPT & 100 & $21.26 \pm 4.644$ & $12-34$ & 130 & $25.79 \pm 21.201$ & $6-118$ & 0.040 & 2.1 \\
\hline Bilirubin & 100 & $0.4621 \pm 0.08211$ & $0.28-0.66$ & 130 & $0.669 \pm 0.25129$ & $0.12-1.8$ & $<0.0001$ & 7.91 \\
\hline$\beta-h C G$ & 100 & $14800 \pm 7284$ & 2655-40835 & 130 & $26700 \pm 17162$ & 5880-92172 & $<0.0001$ & 6.49 \\
\hline
\end{tabular}

There was a statistically significant difference $(p<0.0001)$ in systolic BP, diastolic BP, platelet counts, HDL, LDL, SGOT, SGPT, bilirubin and $\beta$-hCG in the two groups. Student's t-test was applied to calculate the mean of two independent variables 
Table 2: The $p$ value $(p<0.0001)$ should be written in front of mild preeclampsia, severe preeclampsia, and eclampsia

\begin{tabular}{lllll}
\hline & $n$ & Range & Mean $\pm S D$ & $p$-value \\
\hline Normal & 100 & $2655-40835$ & $14803 \pm 7283.5$ & 0.0001 \\
$\begin{array}{l}\text { Mild } \\
\text { preeclampsia }\end{array}$ & 69 & $5880-62455$ & $23023.2 \pm 13727.6$ & \\
$\begin{array}{l}\text { Severe } \\
\text { preeclampsia }\end{array}$ & 31 & $7416-92172$ & $30990.3 \pm 21310.2$ & \\
Eclampsia & 30 & $7591-82202$ & $30680.9 \pm 18171.5$ & \\
\hline
\end{tabular}

The relation of serum $\beta$-hCG with the severity of preeclampsia. The mean level of $\beta$-hCG rose with the severity of hypertension. The values were almost similar in severe preeclampsia and eclampsia

of the disease. That the levels in eclampsia were only comparable to severe preeclampsia and not higher appears to be a paradox, but can be explained by understanding that the onset of a convulsion in a patient of severe preeclampsia leads to immediate referral and definite management of a patient with termination of pregnancy. Understandably, the extent of the pathology in some cases of preeclampsia would be worse than that of eclampsia.

The comparisons of hCG with the blood pressure showed a significant positive correlation with systolic $(\mathrm{p}<0.0001)$ and diastolic $(\mathrm{p}<0.0001)$ blood pressure. There was no correlation between hCG and albuminuria. Studies have not shown any significant correlation of albuminuria with the survival of the patients which can be interpreted as no correlation with the systemic pathology though the level of the blood pressure definitely signifies the severity of the pathological process. This could be extrapolated to mean that the levels of hCG would show a parallel rise with rising levels of blood pressure but not necessarily with the level of albuminuria.

In the laboratory parameters, there was no correlation of hemoglobin with hCG levels (Table 3). One would expect a positive correlation with the level of hCG as preeclampsia leads to hemoconcentration instead of hemodilution. There is a very high prevalence of anemia in our study population which would offset the effect of hemoconcentration. In the end, the level of hemoglobin may tend to be low because of severe anemia and would tend to be high due to hemoconcentration, canceling each other out and not showing any correlation in the overall picture.

We found a significant inverse correlation of hCG levels with the platelet count $(\mathrm{p}=0.0007)$. This would

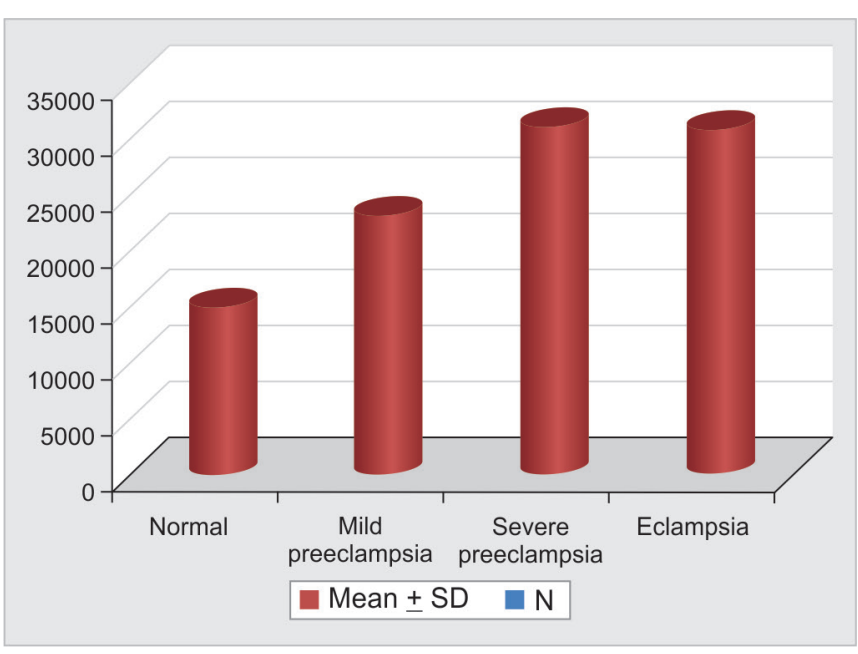

Graph 1: Mean $\beta$-hCG levels in controls and preeclampsia and eclampsia. The Y-axis shows the mean levels of hCG in each group

be expected from the pathogenesis of HELLP syndrome where preeclampsia leads to low platelet counts along with hemolysis and elevated liver enzymes. ${ }^{11,12} \mathrm{~A}$ significant positive correlation was found with AST $(p<0.0001)$, ALT $(p<0.028)$, and serum bilirubin $(\mathrm{p}<0.0001)$, validating the finding of rising levels of $\mathrm{hCG}$ with the progressive pathology of HELLP syndrome, with rising bilirubin signifying the hemolysis occurring with the disease and ALT and AST the derangement of liver enzymes.

A significantly high level of total cholesterol was found in the cases as compared with controls and the HDL levels were significantly lower in the cases as compared with controls. The LDL levels did not show any statistically significant difference between cases and controls. When these levels were correlated with the levels of hCG, a highly significant inverse correlation was found with HDL levels $(p=0.005)$. Apart from this there was no correlation between the levels of total cholesterol and LDL cholesterol with the levels of hCG. Other studies have found a direct correlation between pregnancyinduced hypertension (PIH) and total cholesterol and LDL cholesterol. ${ }^{13}$ High levels of lipids in the blood may lead to oxidative stress which may secondarily lead to endothelial injury, leading to preeclampsia. Our findings suggest the possibility of such a mechanism in the pathogenesis of preeclampsia. The presence of metabolic syndrome may also be a factor leading to preeclampsia. The incidence of PIH may also be related to genetic

Table 3: Correlation of $\beta-h C G$ with the various parameters included in the study

\begin{tabular}{|c|c|c|c|c|c|c|c|c|c|c|c|}
\hline$\beta-h C G$ & $A G E$ & $s B P$ & $D b p$ & $H b$ & Platelets & Cholesterol & $H D L$ & $L D L$ & SGOT & SGPT & Bilirubin \\
\hline Correlation & 0.113 & $0.381^{* *}$ & $0.348^{* *}$ & -0.04 & -0.222 & 0.103 & $-0.185^{* *}$ & -0.092 & $0.312^{* *}$ & $0.145^{\star}$ & $0.291^{* *}$ \\
\hline$p$ & 0.088 & $<0.0001$ & $<0.0001$ & 0.548 & 0.0007 & 0.119 & 0.005 & 0.166 & $<0.0001$ & 0.028 & $<0.0001$ \\
\hline $\mathrm{n}$ & 230 & 230 & 230 & 230 & 230 & 230 & 230 & 230 & 230 & 230 & 230 \\
\hline
\end{tabular}

The table shows a highly significant correlation between $\beta$-hCG and systolic BP, diastolic BP, SGOT and bilirubin $(p<0.0001)$. It also shows a significant correlation of $\beta$-hCG with the platelet count, SGPT and HDL $(p<0.05)$; ${ }^{*}$ Significant $(p<0.05)$, **highly significant $(p<0.0001)$ 


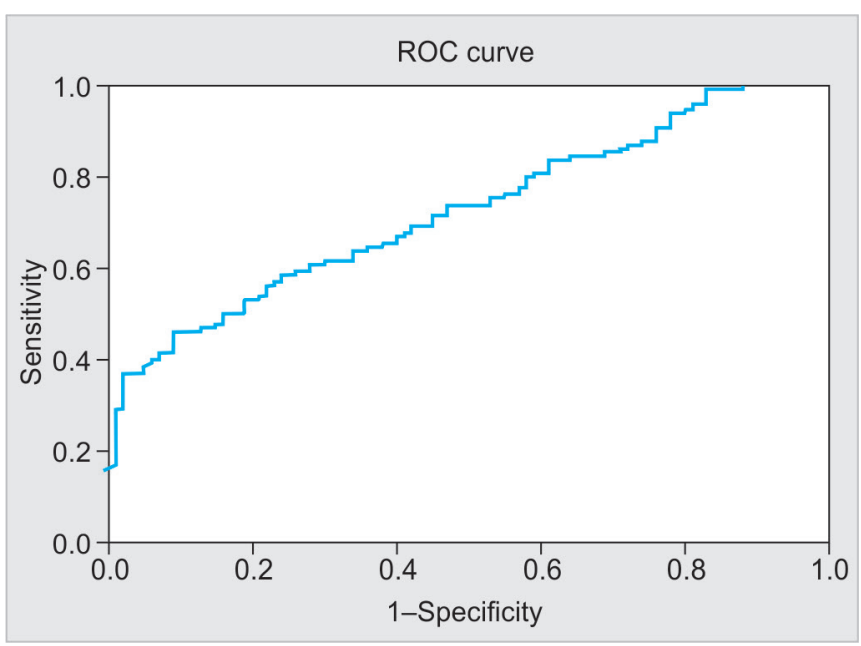

Graph 2: The receiver operating characteristic curve showing the sensitivity and specificity of the levels of $\beta-h C G$ as a diagnostic tool. The receiver operating characteristic curve identified that with cutoff point of $13,485 \mathrm{mlU} / \mathrm{mL}$, the sensitivity and specificity of $\beta$-hCG as diagnostic test in preeclampsia is 75 and $45 \%$ respectively. Area under the curve is $0.724,95 \%$ confidence interval: $0.66-0.788 ; p=0.0001$

and environmental factors. The levels of HCG correlate significantly only with the levels of HDL and showing an inverse relationship. This would be extrapolated to mean that as the protective effect of HDL cholesterol is reduced there is a rise in hCG signifying oxidative stress mediating high levels of hCG. Though the level of total cholesterol was significantly high in the cases as compared with the controls, there was no significant correlation between the hCG levels and the total cholesterol level. hCG may rise with increasing oxidative stress but it does not appear to be a very sensitive indicator of the oxidative stress.

The receiver operating curve plotted for the mean level of hCG and the level of blood pressure showed 75\% sensitivity and $45 \%$ specificity for a cutoff point of 13,485 $\mathrm{mIU} / \mathrm{mL}$ of $\beta$-hCG. The AUC is $0.724,95 \%$ CI: $0.66-0.788$; $\mathrm{p}=0.000$ (Graph 2). This indicates that the levels of serum $\beta$-hCG are neither highly sensitive nor highly specific for diagnosing or predicting the severity of preeclampsia. This poor sensitivity and specificity has been found in other studies. ${ }^{14}$ There would be cases of preeclampsia showing normal levels of $\beta$-hCG and there would also be some normal patients showing high levels of $\beta$-hCG without any pathology. There are studies for the use of the levels of $\beta$-hCG as a predictive test for preeclampsia when done in the 2 nd trimester. ${ }^{15}$

\section{CONCLUSION}

The levels of hCG were significantly high in cases of preeclampsia as compared with normotensive patients and the levels in severe preeclampsia were significantly higher than those of mild preeclampsia as well as normotensive cases. The levels of hCG in eclampsia were comparable with that of severe preeclampsia. The levels of hCG that cannot point to the risk of developing eclampsia is evident from our study. It is not a very sensitive or very specific test for the diagnosis of preeclampsia and as such would have a limited value as a diagnostic tool in these cases due to its poor sensitivity and specificity.

\section{REFERENCES}

1. Leeman L, Fontaine P. Hypertensive disorders of pregnancy. Am Fam Physician 2008 Jul;78(1):93-100.

2. Brodszki J, Länne T, Laurini R, Strevens H, Wide-Swensson D, Marsál K. Vascular mechanical properties and endothelial function in pre-eclampsia with special reference to bilateral uterine artery notch. Acta Obstet Gynecol Scand 2008;87(2): 154-162.

3. Baumwell S, Karumanchi SA. Pre-eclampsia: clinical manifestations and molecular mechanisms. Nephron Clin Pract 2007;106(2):72-81.

4. Mc Master MT, Zhou Y, Fisher SJ. Abnormal placentation and the syndrome of preeclampsia. Semin Nephrol 2004 Nov;24(6):540-547.

5. Fisher SJ. The placental problem: linking abnormal cytotrophoblast differentiation to the maternal symptoms of preeclampsia. Reprod Biol Endocrinol 2004;2:53.

6. Stennett AK, Khalil RA. Neurovascular mechanism of hypertension in pregnancy. Curr Neurovasc Res 2006 May;3(2):131-148.

7. Haram K, Svendsen E, Abildgaard U. The HELLP syndrome: clinical issues and management. A review. BMC Pregnancy Childbirth 2009;9:8.

8. Roiz Hernandez J, de J Cabello-Martinez J, Fernandez-Mejia M. Human chorionic gonadotropin level between 16 and 21 weeks of pregnancy and prediction of preeclampsia. Int J Gynaecol Obstet 2006 Feb;92(2):101-105.

9. Begum Z, Ara I, Tanira S, Keya KA. The association between serum betahuman chorionic gonadotropin and preeclampsia. J Dhaka Med Coll 2014;23(1):89-93.

10. Basirat Z, Barat S, Hajiahmadi M. Serum beta human chorionic gonadotropin level and preeclampsia. Saudi Med J 2006 Jul;27(7):1001-1004.

11. Sultana R, Fazul Karim SM, Atia F, Ferdousi S, Ahmed S. Platelet count in preeclampsia. J Dhaka National Med Coll Hos 2012;18(2):24-26.

12. Annam V, Srinivasa K, Santosh KY, Suresh DR. Evaluation of platelet indices and platelet counts and their significance in preeclampsia and eclampsia. Int J Biol Med Res 2011;2(1): 425-428.

13. Mallick MP, Ray S, Medhi R, Bisai S. Elevated serum $\beta$ hCG and dyslipidemia in second trimester as predictors of subsequent pregnancy induced hypertension. Bangladesh Med Res Counc Bull 2014 Dec;40(3):97-101.

14. Yousefnejad K, Moslemizadeh N. Serum B-hCG levels in diagnosis and management of preeclampsia. J Med Sci 2008;8(8):722-727.

15. Dayal M, Gupta P, Varma M, Ghosh UK, Bhargava A. Role of second trimester maternal serum markers as predictor of preeclampsia. J Obstet Gynaecol India 2011 Feb;61(1):38-41. 